\title{
Accumulative copy number increase of MET drives tumor development and histological progression in a subset of ovarian clear-cell adenocarcinomas
}

\author{
Sohei Yamamoto ${ }^{1}$, Hitoshi Tsuda ${ }^{2}$, Kosuke Miyai ${ }^{1}$, Masashi Takano ${ }^{3}$, Seiichi Tamai ${ }^{4}$ \\ and Osamu Matsubara ${ }^{1}$ \\ ${ }^{1}$ Department of Basic Pathology, National Defense Medical College, Tokorozawa, Japan; ${ }^{2}$ Pathology and \\ Clinical Laboratory Division, National Cancer Center Hospital, Chuo-ku, Tokyo, Japan; ${ }^{3}$ Department of \\ Obstetrics and Gynecology, National Defense Medical College, Tokorozawa, Japan and ${ }^{4}$ Department of \\ Clinical Laboratory, National Defense Medical College Hospital, Tokorozawa, Japan
}

Our previous study demonstrated that, among ovarian carcinomas, amplification of the MET gene and overexpression of MET specifically and commonly occur in clear-cell adenocarcinoma histology. This study was conducted to address how these alterations contribute to development and progression of this highly chemoresistant form of ovarian cancer. We histologically reviewed 21 previously described MET amplificationpositive clear-cell adenocarcinoma cases, and selected 11 tumors with synchronous endometriosis and 2 tumors with adjacent clear-cell adenofibroma (CCAF) components. Using double in situ hybridization and immunohistochemistry, copy number alterations of the MET gene and levels of MET protein expression were analyzed in these putative precursor lesions and the corresponding invasive carcinoma components in this selected cohort. All of the non-atypical precursor lesions analyzed (ie, non-atypical endometrioses and the benign CCAFs) were negative for MET gain. However, low-level ( $\geq 3$ MET copies in $\geq 10 \%$ and $\geq 4$ MET copies in $10-40 \%$ of tumor cells) gain of MET was detected in $4(40 \%)$ of the 10 atypical endometrioses and 1 of the 2 borderline CCAFs. Moreover, high-level ( $\geq 4$ MET copies in $\geq 40 \%$ of tumor cells) gain of MET were detected in five $(50 \%)$ of the atypical endometrioses. In $4(31 \%)$ of the 13 cases enrolled, intratumoral heterogeneity for MET gain was documented in invasive carcinoma components, wherein all the relatively differentiated carcinoma components showed low-level gain of MET and all the corresponding poorly differentiated carcinomas showed high-level gain. The overall incidence of MET overexpression gradually increased from the precursors of non-atypical form (0\%), through those of atypical form $(67 \%)$ and the relatively differentiated carcinoma components $(92 \%)$, to the poorly differentiated carcinoma components (100\%). These results suggest that accumulative MET gene copy number alterations causing MET overexpression are associated with higher tumor grade and might drive the development and progression of the MET amplification-positive ovarian clear-cell adenocarcinoma.

Modern Pathology (2012) 25, 122-130; doi:10.1038/modpathol.2011.143; published online 7 October 2011

Keywords: clear cell adenocarcinoma; c-Met; gene amplification; in situ hybridization; MET; ovary; progression

Perhaps, the most important characteristic of any tumor is the combination of genetic alterations that underlie its development and drive its progression.

Correspondence: Dr H Tsuda, MD, Pathology and Clinical Laboratory Division, National Cancer Center Hospital, 5-1-1 Tsukiji, Chuo-ku, Tokyo 104-0045, Japan.

E-mail: hstsuda@ncc.go.jp

Received 2 May 2011; revised 26 July 2011; accepted 3 August 2011; published online 7 October 2011
The search for new target genes for anticancer therapies has become a discrete field of translational research. MET, a unique member of the receptor tyrosine kinase family, has attracted much attention in the recent years, representing an intriguing target for cancer therapy, although this has not yet been established in a clinical setting. The MET protooncogene, located on chromosome $7 \mathrm{q} 31$, encodes the MET kinase that is composed of three functional domains, including the ligand-binding domain, 
regulatory juxtamembrane domain, and the receptor tyrosine kinase domain. ${ }^{1}$ Physiologically, when its ligand, namely, hepatocyte growth factor (HGF), binds to it, the MET receptor undergoes dimerization and autophosphorylation at specific tyrosine residues within the cytoplasmic domain, creating docking sites for intracellular signal transducers that activate the Ras-mitogen-activated protein kinase pathway, and the phosphatidylinositol 3-kinaseAKT-mTOR signaling pathway, as well as several other pathways. ${ }^{1,2}$

In contrast to the non-neoplastic cells, in the tumor cells, MET can be activated in a ligand-independent manner through activating mutation, amplification, and overexpression of the MET gene. ${ }^{1-3}$ However, to date, it is thought that somatic mutation of MET is a rare event in the sporadic primary carcinomas of adults, including ovarian carcinomas, ${ }^{4,5}$ with papillary carcinoma of the kidney being an exception. ${ }^{6}$ On the other hand, MET amplification was identified in $5-10 \%$ of gastric cancers, ${ }^{7-9} 4 \%$ of esophageal cancers, ${ }^{10} 3-4 \%$ of lung cancers, ${ }^{11,12}$ and $10 \%$ of colorectal cancers. ${ }^{13}$

Our recent study identified that MET overexpression and gene amplification were commonly detected in ovarian clear-cell adenocarcinoma, with their frequencies at 22 and $24 \%$, respectively. ${ }^{14}$ On the other hand, such alterations were extremely rare in the non-clear cell histological subtypes of ovarian carcinoma (ie, serous, endometrioid, and mucinous adenocarcinomas). ${ }^{14}$ In that study, MET overexpression was associated with a worse prognosis of the clear-cell adenocarcinoma patients, and MET gene amplification was correlated with MET overexpression and poorly differentiated histology of tumors. ${ }^{14}$ These findings suggested that among ovarian carcinomas, MET proto-oncogene is specifically involved in clear-cell adenocarcinoma by means of gene amplification. However, it remains unclear how these alterations contribute to tumor development and progression of this carcinoma type.

Clear-cell adenocarcinoma has been recognized to be a highly chemo-resistant form of ovarian cancer. ${ }^{15-19}$ Although little is known about the molecular genetic alterations underlying tumor development, a hypothesis of multi-step tumorigenesis of ovarian clear-cell adenocarcinoma, starting with histologically benignappearing precursor lesions (ie, endometriosis and benign clear-cell adenofibroma (CCAF)), progressing to their atypical counterparts (ie, atypical endometriosis and borderline CCAF), and ultimately to clearcell adenocarcinoma has been proposed and widely considered. ${ }^{20-26}$

In this study, to unmask the timing of MET alterations in the development and progression of ovarian clear-cell adenocarcinoma, we selected 13 clear-cell adenocarcinoma cases that were double in situ hybridization-positive cases, as determined by our previous series, ${ }^{14}$ and were in a group of carcinomas with adjacent putative precursor lesions. Using double in situ hybridization and immunohistochemistry, the enrolled cases were analyzed for MET gene copy number alterations and MET protein expression in terms of their intratumoral heterogeneity. Specifically, MET alterations were examined in the benign-appearing putative precursor lesions (ie, non-atypical endometriosis and benign CCAF), their atypical forms (atypical endometriosis and borderline CCAF), and the corresponding invasive carcinoma components. Moreover, the invasive carcinoma components were divided into the two histological categories (ie, carcinomas of poorly differentiated histology VS those of relatively differentiated histology), and the heterogeneity regarding MET alterations in a carcinoma was also assessed. It was anticipated that this information would not only lead to a better understanding of the development of ovarian clearcell adenocarcinoma, but also provide insight into the potential treatment options for this highly chemo-resistant malignancy.

\section{Materials and methods}

\section{Patients and Tissue Samples}

Hematoxylin- and eosin-stained sections from 21 clear-cell adenocarcinoma cases, in which our previous double in situ hybridization analysis revealed high-level polysomy or gene amplification of MET in the invasive carcinoma components, ${ }^{14}$ were histologically reviewed. From these 21 cases, based on the histopathological criteria described previously, ${ }^{21-24}$ 11 tumors with synchronous endometriotic lesions (endometriosis-associated carcinomas) and 2 tumors with adjacent CCAF components (adenofibromaassociated carcinomas) were identified. These 13 cases were retrieved from the files of the Department of Clinical Laboratory, National Defense Medical College Hospital, Japan; all patients had undergone surgical resection between 1987 and 2006, none had undergone chemotherapy or radiation therapy before surgery, and all specimens were formalin-fixed and paraffin-embedded tissue sections. Clinical staging of disease was done according to the International Federation of Gynecology and Obstetrics system. Of the 11 cases with endometriosis-associated carcinoma, $8(73 \%)$ were stage I, 1 (9\%) was stage II, $1(9 \%)$ was stage III, and $1(9 \%)$ was stage IV. Of the two cases with adenofibroma-associated carcinoma, one was stage I, and another was stage II. The research protocol was approved by the ethics committee of the National Defense Medical College, Tokorozawa, Japan.

On the basis of previously described histological criteria for 'atypical endometriosis', ${ }^{21-23}$ endometriotic lesions adjacent to clear-cell adenocarcinoma were classified into non-atypical and atypical forms. Of the 11 endometriosis-associated cases, 9 had both non-atypical and atypical endometriosis, 1 had nonatypical endometriosis only, and 1 had atypical endometriosis only. Therefore, 10 lesions of non-atypical 
endometriosis, 10 lesions of atypical endometriosis, and 11 clear-cell adenocarcinomas containing synchronous endometriosis were analyzed by double in situ hybridization and immunohistochemistry.

Both the adenofibroma-associated carcinomas had components of benign CCAF and borderline CCAF. The histopathological criteria for 'benign-' and 'borderline-' CCAF were described in the previous report. ${ }^{24}$

\section{Poorly Differentiated Histology in Clear-Cell Adenocarcinoma}

To further assess the intra-tumoral heterogeneity of MET alterations in invasive carcinoma components, we established criteria for evaluating poorly differentiated carcinoma histology, and divided the carcinoma components into two groups: poorly differentiated histology and relatively differentiated histology. Details of the criteria for poorly differentiated histology of clear-cell adenocarcinoma were described in our previous series. ${ }^{14}$ Briefly, when tumor cells grew as solid masses, cords, or individual tumor cells, infiltrating towards the surrounding stromal tissue without easily discernible glandular differentiation, these were defined as exhibiting poorly differentiated histology. On the other hand, tumor cells whose growth was noted to be typically tubulocystic, papillary, or a combination of these (tubulopapillary), were defined as having a relatively differentiated histology of clear-cell adenocarcinoma. Of the 13 cases enrolled, 10 cases had both the relatively differentiated and the poorly differentiated histological components, and other 3 had the relatively differentiated histological component only. Therefore, 13 lesions of the relatively differentiated clear-cell adenocarcinoma component and 10 lesions of the poorly differentiated clear-cell adenocarcinoma component were analyzed by double in situ hybridization and immunohistochemistry.

\section{Bright-Field Double In Situ Hybridization}

A BenchMark XT automated slide processing system (Ventana Medical Systems, Tucson, AZ, USA) was used for the optimization of the double in situ hybridization assay for copy number alteration of MET. Technical details for this assay were described in the previous reports. ${ }^{14,27}$ Briefly, for MET detection, the INFORM MET DNA Probe (Ventana), a dinitrophenyl (DNP)-labeled probe, was applied to the tissue sections, denatured at $95^{\circ} \mathrm{C}$, and hybridized at $52{ }^{\circ} \mathrm{C}$ for $6 \mathrm{~h}$. After washing, the tissue sections were incubated with monoclonal rabbit anti-DNP antibody (Ventana) for $20 \mathrm{~min}$, and then with horseradish peroxidise-conjugated goat antirabbit antibody for $16 \mathrm{~min}$ at $37^{\circ} \mathrm{C}$. The metallic silver deposit for MET ISH signal was developed using the ultraView SISH Detection Kit (Ventana). For CEN7 detection, the INFORM Chromosome 7 Probe (Ventana), a DNP-labelled oligoprobe, was applied to the tissue sections, denatured at $95^{\circ} \mathrm{C}$, and hybridized at $44^{\circ} \mathrm{C}$ for $2 \mathrm{~h}$. Then, after washing, the tissue sections were incubated with monoclonal rabbit anti-DNP antibody for $20 \mathrm{~min}$, and then with an alkaline phosphatase-conjugated goat anti-rabbit antibody for $12 \mathrm{~min}$ at $37^{\circ} \mathrm{C}$. The signal for CEN7 was visualized with a Fast-Red and naphthol phosphate reaction, using an ultraView Red ISH Detection Kit. Finally, hematoxylin counterstaining was performed.

\section{Immunohistochemistry}

For MET protein detection, a BenchMark XT automated slide processing system was also used, as described previously. ${ }^{14}$ The primary antibody used was a rabbit monoclonal antibody against the carboxyl region of the transmembrane human c-Met protein (CONFIRM anti-Total c-MET (SP44), ready for use; Ventana). The immunoreaction was visualized using an ultraView DAB Detection Kit (Ventana) according to the manufacturer's instructions. ${ }^{27}$ Endothelial cells in the tumor tissues served as positive controls, and sections without the primary antibody were used as negative controls.

\section{Interpretation of the Data}

For double in situ hybridization analysis, the numbers of dark brown and red dot signals, corresponding to the copies of MET and those of CEN7, respectively, were counted in 80 inter-phase tumor cell nuclei by using a $\times 100$ oil immersion objective lens. All cases were arranged in three categories as follows: no gain $(\leq 2 M E T$ copies in $>90 \%$ of cells), low-level gain ( $\geq 3 \mathrm{MET}$ copies in $\geq 10 \%$ of cells and $\geq 4$ copies of MET in $<40 \%$ of cells), and high-level gain (status of high polysomy ( $\geq 4$ copies of $M E T$ in $\geq 40 \%$ of cells) or gene amplification (presence of tight gene clusters, a MET/CEN7 ratio per cell of $\geq 2$, or $\geq 15$ copies of the MET gene in $\geq 10 \%$ of cells examined)), by using the previously described criteria. ${ }^{14,28}$

The intensity of the immunoreaction was scored using a four-tier system with the same criteria used for assessing HER2/neu immunoreactions in breast cancer (the HercepTest criteria) as follows: ${ }^{29}$ negative, no discernible staining or background type staining; $1+$, definite cytoplasmic staining and/or equivocal discontinuous membrane staining; $2+$, unequivocal membrane staining with mild to moderate intensity; $3+$, strong and complete membrane staining. MET overexpression was defined as moderate $(2+)$ or strong $(3+)$, when complete membrane staining was observed in at least $10 \%$ of the cells of interest.

\section{Statistical Analysis}

Statistical analyses were performed using StatMate III software (ATMS, Tokyo, Japan). Comparisons 
between parameters were computed by the $\chi^{2}$-test. Differences at $P<0.05$ were considered statistically significant.

\section{Results}

The results of double in situ hybridization and immunohistochemistry for detection of MET alterations are given in Table 1.

\section{Copy Number Alterations of the MET Gene and Overexpression of MET Frequently Occur in the Putative Precursor Lesions, Especially in their Atypical Forms}

All the 10 non-atypical endometrioses examined were found to exhibit no gain of $M E T$ by double in situ hybridization assay, and all showed weak immunoreactions for MET, with scores of $1+$ (Figure 1). Of the 10 atypical endometrioses, $1(10 \%), 4(40 \%)$, and $5(50 \%)$ lesions were defined as exhibiting no gain, low-level gain, and high-level gain of MET, respectively (Figure 1). All the atypical endometriosis showing high-level gain of the MET gene and three of the four atypical endometrioses showing low-level gain were defined as exhibiting MET overexpression by immunohistochemistry (Figure 1). The remaining one atypical endometriosis showed no gain of MET, with a weak (score $1+$ ) immunoreaction for MET.

Both of benign CCAFs showed no gain of MET and a weak (score 1+) immunoreaction for MET (Figure 2). One borderline CCAF showed low-level gain of MET (Figure 2), and another borderline CCAF showed no gain. Weak immunoreaction for MET was noted in these two borderline CCAF components, but MET overexpression was not observed (Figure 2).

Heterogeneity of MET alterations in the invasive carcinoma components is common (Table 1).

Of the five endometriosis-associated cases wherein adjacent atypical endometrioses harbored a high-level gain of $M E T$ (case numbers 2, 4, 8, 9, and 10), all the corresponding carcinoma components examined, regardless of the extent of tumor differentiation (relatively differentiated vs poorly differentiated components), also showed high-level gain of MET and overexpression of MET. In case number 9, although both the relatively differentiated and poorly differentiated carcinoma components were defined as exhibiting a high-level gain, only the latter component showed true amplification of MET (Figure 3).

Of the five tumors in which the adjacent precursors (ie, atypical endometriosis and borderline CCAF) showed low-level gain of MET (case numbers $1,3,5,6$, and 13), intra-tumoral heterogeneity of the copy number alterations of the MET gene was detected in two carcinomas (case numbers 5 and 6). In these two cases, carcinoma components of relatively differentiated histology were defined as exhibiting low-level gain of $M E T$, whereas those of poorly differentiated histology exhibited highlevel gain.

Of the other three tumors in which double in situ hybridization analysis did not reveal copy number alterations of $M E T$ in the adjacent precursor lesions (case numbers 7, 11, and 12), intra-tumoral heterogeneity of the copy number alterations of MET was detected in two carcinomas (case numbers 7 and 11).

Table 1 MET alterations in ovarian clear-cell adenocarcinomas and their co-existing putative precursor lesions, as detected by immunohistochemistry and double in situ hybridization

\begin{tabular}{|c|c|c|c|c|c|c|c|c|c|}
\hline \multirow{3}{*}{$\begin{array}{l}\text { Case no. } \\
\text { (adjacent } \\
\text { precursor lesion) }\end{array}$} & \multirow[t]{3}{*}{$\begin{array}{l}\text { Clinical stage } \\
\text { of disease }^{\mathrm{a}}\end{array}$} & \multicolumn{2}{|c|}{$\begin{array}{c}\text { Precursor lesion } \\
\text { without atypia }\end{array}$} & \multicolumn{2}{|c|}{$\begin{array}{l}\text { Precursor lesion } \\
\text { with atypia }\end{array}$} & \multicolumn{4}{|c|}{ Carcinoma } \\
\hline & & \multirow[b]{2}{*}{$I H C$} & \multirow[b]{2}{*}{ DISH } & \multirow[b]{2}{*}{$I H C$} & \multirow[b]{2}{*}{ DISH } & \multicolumn{2}{|c|}{$\begin{array}{c}\text { Relatively } \\
\text { differentiated }\end{array}$} & \multicolumn{2}{|c|}{$\begin{array}{c}\text { Poorly } \\
\text { differentiated }\end{array}$} \\
\hline & & & & & & $I H C$ & DISH & $I H C$ & DISH \\
\hline 1 (Endometriosis) & Ic & $1+$ & No gain & $3+$ & Low gain & $3+$ & High gain & - & - \\
\hline 2 (Endometriosis) & Ic & $1+$ & No gain & $2+$ & High gain & $3+$ & High gain & $2+$ & High gain \\
\hline 3 (Endometriosis) & Ia & $1+$ & No gain & $1+$ & Low gain & $2+$ & High gain & - & - \\
\hline 4 (Endometriosis) & IV & $1+$ & No gain & $2+$ & High gain & $2+$ & High gain & $2+$ & High gain \\
\hline 5 (Endometriosis) & IIIc & $1+$ & No gain & $2+$ & Low gain & $2+$ & Low gain & $3+$ & High gain \\
\hline 6 (Endometriosis) & Ic & $1+$ & No gain & $2+$ & Low gain & $3+$ & Low gain & $3+$ & High gain \\
\hline 7 (Endometriosis) & Ia & $1+$ & No gain & $1+$ & No gain & $2+$ & Low gain & $3+$ & Amplified \\
\hline 8 (Endometriosis) & Ia & $1+$ & No gain & $2+$ & High gain & $2+$ & High gain & $3+$ & High gain \\
\hline 9 (Endometriosis) & Ia & $1+$ & No gain & $2+$ & High gain & $3+$ & High gain & $3+$ & Amplified \\
\hline 10 (Endometriosis) & Ic & - & - & $2+$ & High gain & $2+$ & High gain & - & -1 \\
\hline 11 (Endometriosis) & IIc & $1+$ & No gain & - & - & $1+$ & Low gain & $2+$ & High gain \\
\hline 12 (CCAF) & Ic & $1+$ & No gain & $1+$ & No gain & $2+$ & High gain & $2+$ & High gain \\
\hline 13 (CCAF) & IIc & $1+$ & No gain & $1+$ & Low gain & $2+$ & High gain & $3+$ & High gain \\
\hline
\end{tabular}

Abbreviations: CCAF, clear-cell adenofibroma; DISH, double in situ hybridization; IHC, immunohistochemistry.

${ }^{\mathrm{a}}$ Clinical stages of disease defined by International Federation of Gynecology and Obstetrics. 

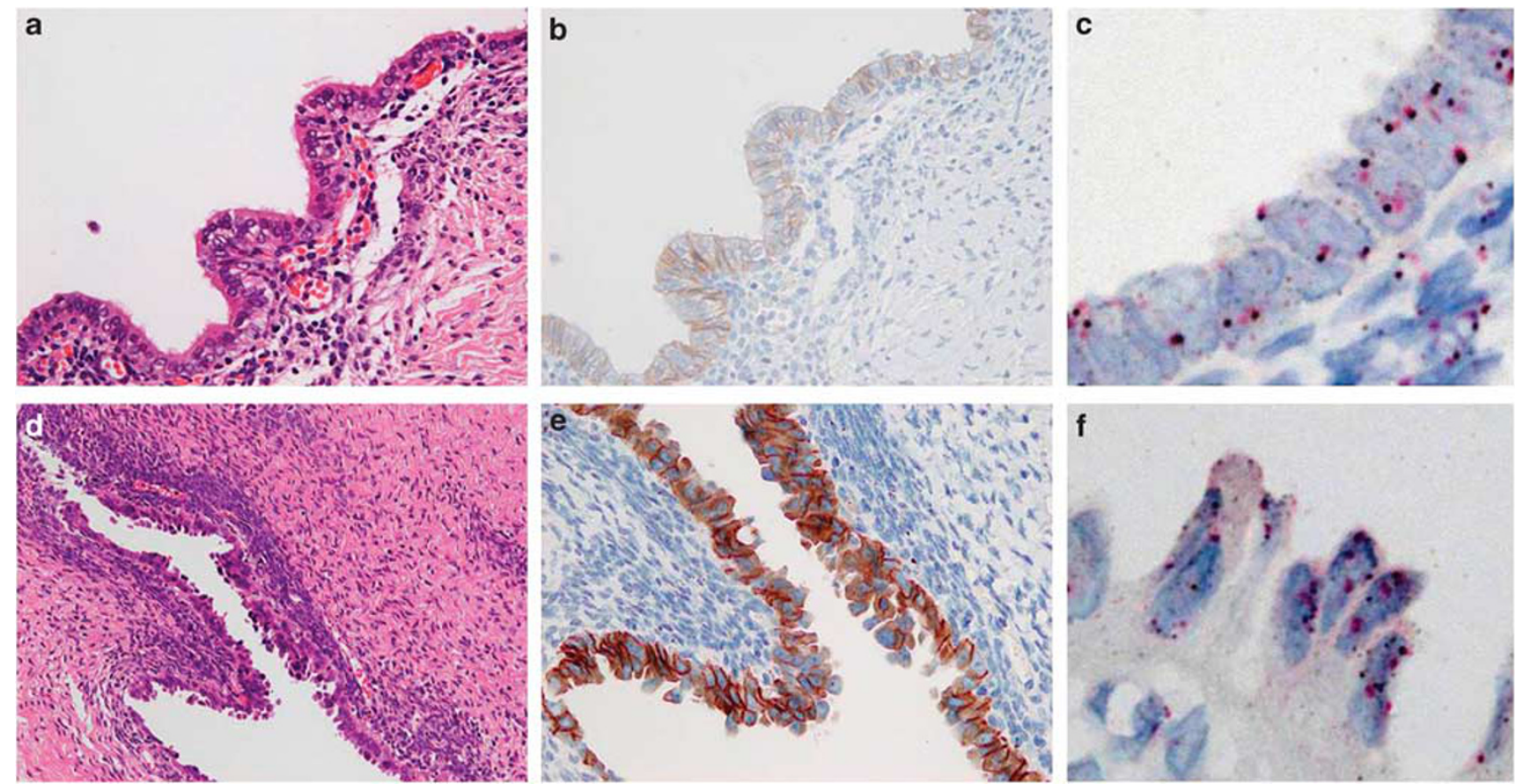

Figure 1 MET alterations in non-atypical endometriosis (a-c) and atypical endometriosis (d-f) synchronous with ovarian clear-cell adenocarcinoma. (a) Representative histological features of non-atypical endometriosis. The endometriotic epithelia lack evident cytological or structural atypia. (b) Weak but definite cytoplasmic immunoreaction is noted in the endometriotic epithelium. According to the described criteria, this lesion was classified as weak (score 1+) for MET immunoreaction. (c) Most of the epithelial cells in this photomicrographs show one to two dark brown (corresponding to the MET gene) and red (corresponding to the centromeric DNA region on chromosome 7 (CEN7)) signals. According to the described criteria, this lesion was defined as exhibiting no gain of the MET gene. (d) Representative histological features of an atypical endometriosis. Endometriotic epithelia show moderate degrees of cytological atypia and cellular stratification. (e) Continuous membrane immunoreaction with moderate intensity is noted in this atypical endometriosis. According to the described criteria, this lesion was classified as exhibiting MET overexpression (score 2+). (f) Atypical endometriosis classified as showing high-level gain of the MET gene. About half of the epithelial cells in this photomicrograph show four pairs of MET and CEN7 signals. (a and d) HE stain, original magnification $\times 400$ for (a) and $\times 200$ for (d). (b and e) Immunoperoxidase stain, original magnification $\times 400$ for both. (c and f) Double in situ hybridization assays.

In these two cases, carcinoma components of relatively differentiated histology were defined as exhibiting low-level gain of MET, whereas those of poorly differentiated histology exhibited high-level gain. In case number 7 , the poorly differentiated carcinoma component showed true amplification of the MET gene. Both the relatively differentiated and poorly differentiated carcinoma components in the remaining one case (case number 12) showed a highlevel gain of the MET gene.

The overall incidence of high-level gain of the $M E T$ gene was found to gradually increase from the precursors of non-atypical form $(0 \%)$, through those of atypical form $(42 \%)$ and the relatively differentiated carcinoma components $(69 \%)$, to the poorly differentiated carcinoma components $(100 \%)$. Consequently, statistical analyses revealed significant differences in the frequency between the nonatypical and atypical precursors $(P=0.019)$, and between the atypical precursors and poorly differentiated carcinoma components $(P=0.0046)$. Moreover, the overall incidence of MET overexpression was also observed to gradually increase from the precursors of non-atypical form ( $0 \%)$, through those of atypical form (67\%) and the relatively differentiated carcinoma components (92\%), to the poorly differentiated carcinoma components $(100 \%)$. Consequently, statistical analyses revealed significant differences in the frequency between the nonatypical and atypical precursors $(P=0.0007)$ or invasive carcinoma components $(P<0.0001)$.

\section{Discussion}

The main findings of our investigation can be summarized as follows: (1) MET overexpression and copy number alterations were not detected in the non-atypical forms of precursor lesions, such as non-atypical endometriosis and benign CCAF; (2) these alterations were frequently detected in the atypical forms of precursors (ie, atypical endometriosis and borderline CCAF) in the MET amplification-positive ovarian clear-cell adenocarcinoma, and some of these lesions already harbor a high-level gain of MET; (3) intra-tumoral heterogeneity of MET alterations in invasive carcinoma components is common; and (4) rates of the MET alterations were 

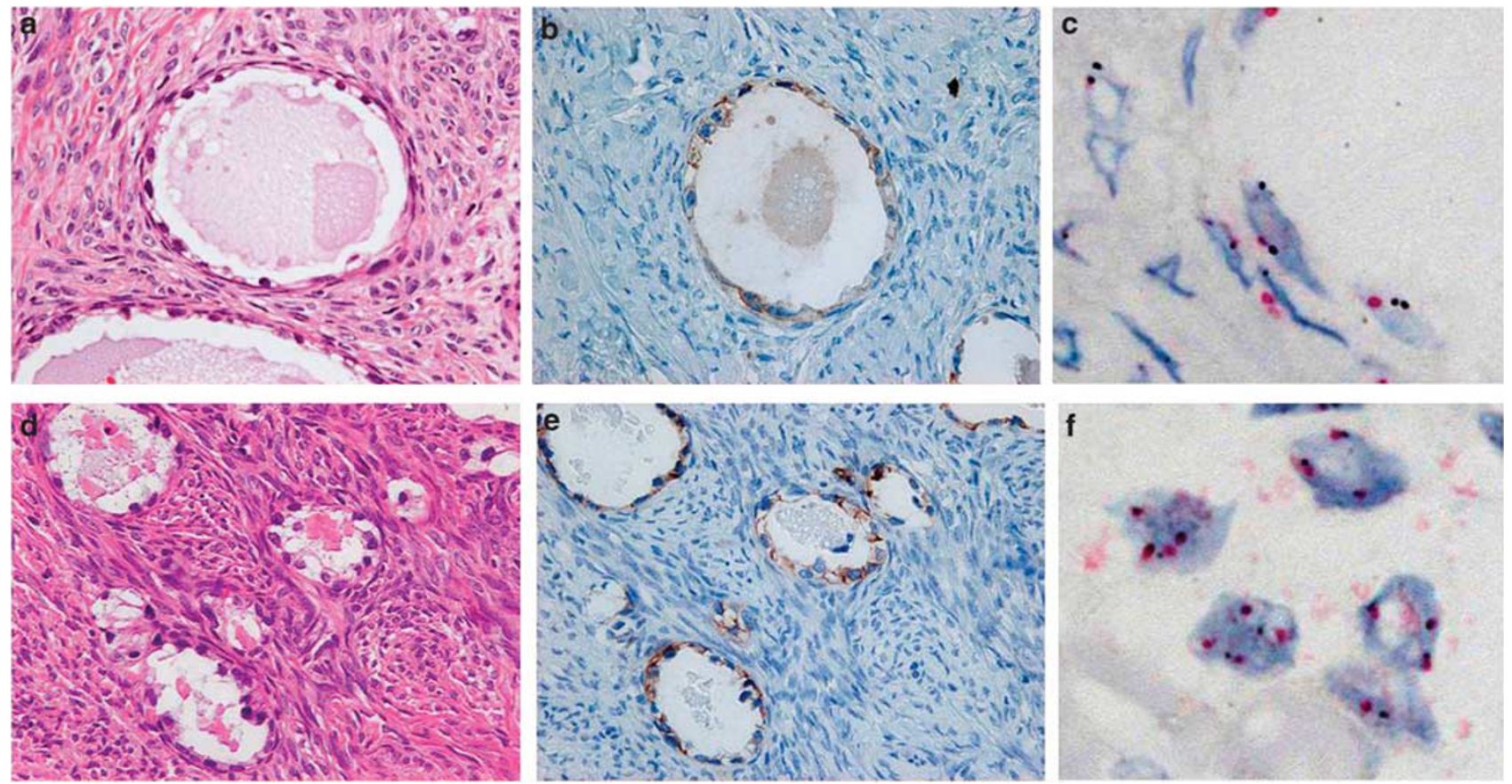

Figure 2 MET alterations in the benign clear-cell adenofibroma (CCAF) (a-c), and the borderline CCAF (d-f) adjacent to ovarian clear-cell adenocarcinoma. (a) A case of benign CCAF, lacking cytological or structural atypia. (b) Weak but definite cytoplasmic immunoreaction is noted in the epithelial component. This lesion was assigned a score of $1+$ for MET immunoreaction. (c) The epithelial cells of CCAF showing one to two pairs of MET (dark brown) and CEN7 (red) signals. This lesion was defined as exhibiting no gain of the MET gene. (d) A case of borderline CCAF. Epithelial cells show a moderate degree of cytological atypia. (e) Weak (score $1+$ ) immunoreactions were noted in the epithelial components. (f) The epithelial cells of borderline CCAF showing three to four pairs of MET and CEN7 signals. According to the described criteria, this component was defined as exhibiting a low-level gain of MET. (a and d) Hematoxylin and eosin (HE) stain, original magnification $\times 400$ for both. (b and e) Immunoperoxidase stain, original magnification $\times 400$ for both. (c and $\mathbf{f}$ ) Double in situ hybridization assays.

higher in the poorly differentiated carcinoma histology than in the relatively differentiated histology of ovarian clear-cell adenocarcinomas. These findings suggest that MET alterations occur as an early event in carcinogenesis of the MET amplification-positive clear-cell adenocarcinoma of ovary, and that, these alterations might drive the development and progression in a subset of ovarian clear-cell adenocarcinoma. This is the first report to demonstrate the evidence of MET copy number abnormalities and MET overexpression in the putative precursor lesions of ovarian clear-cell adenocarcinoma.

Although our analysis failed to reveal MET overexpression in the non-atypical precursor lesions, all these lesions showed weak levels of MET-associated immunoreactions. Small amounts of MET protein expression were detected in the normal Mullerian epithelium (ie, endocervical glands, endometrium, and ovarian surface epithelium). ${ }^{30}$ Moreover, the pathophysiology is still elusive. It has been generally believed that HGF retains a multifunctional role (ie, functions in mitogenesis, cell migration, angiogenesis, and morphogenesis) in the pathogenesis of the pelvic solitary endometriosis, in combination with peritoneal macrophages and ovarian steroids. ${ }^{31}$ Therefore, it could be suggested that in (non-atypical) endometrioses and benign CCAFs, low-level expression of MET (ie, expression level scored as $1+$ in the present study), in combination with the locally secreted HGF, might be related to cellular differentiation, for example, to the Mullerian epithelial differentiation, and not be directly associated with cancer development and progression.

In our previous report, although a strong correlation between MET overexpression and double in situ hybridization positivity was statistically supported, there were some discrepancies between the results of immunohistochemistry and double in situ hybridization analyses. However, in the present study, all carcinoma components and atypical precursor lesions harboring a high-level gain of MET demonstrated MET overexpression. These differences in the results obtained were probably because of the different sample types used for analyses; wholesection samples were used in the present series, whereas two tissue microarray cores per case were used in the previous report, and the latter might only represent small parts of the carcinoma. In addition, six $(67 \%)$ of the nine lesions with lowlevel gain of MET showed MET overexpression, and none of the lesions with no gain of MET showed MET overexpression. Therefore, it is highly likely that MET gene amplification is indeed an important 

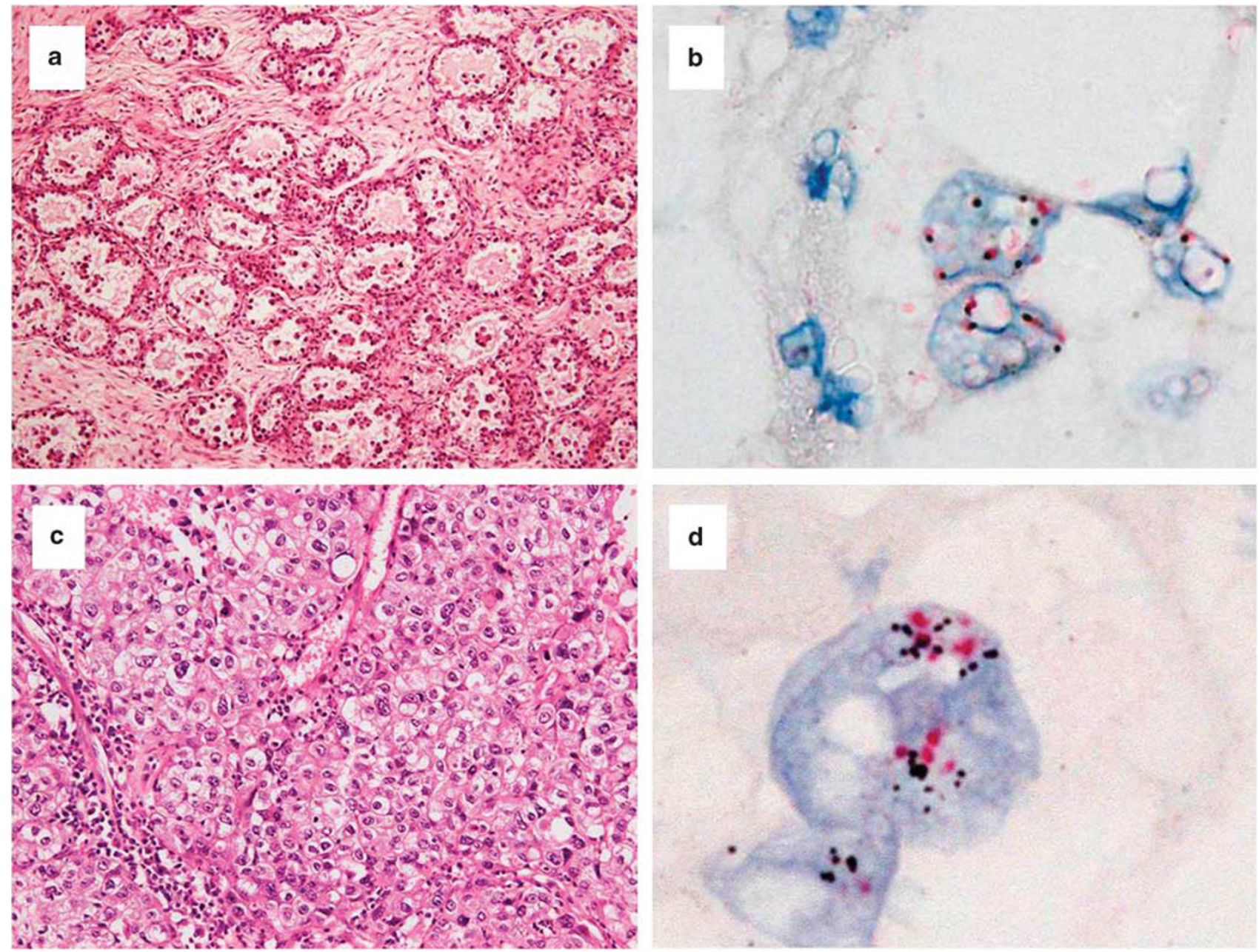

Figure 3 Intra-tumoral heterogeneity of $M E T$ gain in an ovarian clear-cell adenocarcinoma. (a) Representative histology of a relatively differentiated carcinoma component in case number 9. Carcinoma cells growing infiltrating tubular architectures composed of mediumsize and uniformly rounded glands of tumor cells. Some glands of tumor cells are combined with small papillary architectures, exhibiting a 'tubulopapillary' feature. (b) In this microphotograph, the carcinoma cells of the relatively differentiated histology show seven or four pairs of MET (dark brown) and CEP7 (red) signals. This lesion was defined as exhibiting a high gain of MET, but not true amplification. (c) A poorly differentiated histological component in case number. Tumor cells growing by solid sheets of carcinoma cells, without distinct glandular formations. (d) True gene amplification in tumor cells of poorly differentiated histology in case number. In the focused tumor cell, MET signals form gene clusters, and the ratio of MET:CEN7 per cell is clearly more than 2. (a and c) Hematoxylin and eosin (HE) stain, original magnification $\times 100$ for (a) and $\times 200$ for (c). (b and d) Double in situ hybridization assays.

mechanism for MET overexpression in ovarian clearcell carcinogenesis.

What are the potential implications of these findings with regard to treatment of ovarian clearcell adenocarcinoma with MET inhibitors? In recent years, the evidence accumulated suggests that MET is an exciting and novel drug target for the treatment of MET-overexpressing ovarian cancers, because of the success observed in vitro and in vivo. ${ }^{32,33}$ Various parameters have been suggested to be the predictors of the response to MET kinase inhibitors, including strong expression, as seen, for example, in lung cancers, and gene amplification, as seen in lung and gastric cancers. ${ }^{12,34-37}$ Taken together with our present data, MET appears to be an important therapeutic target in the treatment of ovarian clear-cell adenocarcinomas with MET alterations, justifying the exploration of anti-MET treatment strategies. Moreover, as HGF is the only known ligand for the MET receptor, neutralization of HGF using ribozymes $^{38}$ or antagonistic fragments such as NK4 ${ }^{39}$ and neutralizing antibodies ${ }^{40}$ may also be the potentially attractive compounds for inhibiting HGF/MET signaling.

Identification of the molecular mechanisms responsible for aggressive tumor behavior is also important for the development of potential new treatment strategies. Although, until recently, no histological features predictive of the clinical outcome in ovarian clear-cell adenocarcinoma patients have been agreed upon, we recently reported that clear-cell adenocarcinomas with poorly differentiated histology, 
accounting for $33 \%$ of the cases studied, had significantly worse outcomes than those with well (not poorly)-differentiated histology, both in earlystage and advanced-stage diseases. ${ }^{41}$ Moreover, the presence of poorly differentiated histology was highly associated with resistance of the tumor to postoperative platinum-based chemotherapy. ${ }^{41}$ These findings suggested that tumors with poorly differentiated histology can be regarded as a high-grade subtype of ovarian clear-cell adenocarcinoma. In the current study, intratumoral heterogeneity for MET alterations were found to be common in invasive carcinoma components, wherein MET alterations were usually more evident in the poorly differentiated histology than in the relatively differentiated carcinoma components, suggesting that MET alterations may be associated with the histological progression of MET amplification-positive ovarian clear-cell adenocarcinoma. Therefore, MET gains may provide a carcinoma component with evolutionary advantages, favoring its transformation into higher grade and aggressive subtypes of this carcinoma type. Considering the overall data available, it can be stated that MET should be examined as a potential target for treatment of ovarian clear-cell adenocarcinoma, especially those of the aggressive, high-grade, and chemo-resistant subgroup.

In conclusion, our data suggest that copy number alterations of MET and MET overexpression are critical steps in the early development of METamplified and high-grade ovarian clear-cell adenocarcinomas, and that MET amplification, in particular, may prove to be an excellent biomarker of histological progression of this carcinoma. These results contribute to the understanding of the pathogenesis of clear-cell adenocarcinoma and support the development of targeted therapies that inhibit MET activation.

\section{Acknowledgements}

This work was supported in part by a grant-in-aid for cancer research from the Ministry of Health, Labor, and Welfare, Japan (HT), and by a grant from the Foundation for Promotion of Cancer Research (SY and HT). We are grateful to Eiko Munechika, MT, Roche Diagnostics, Tokyo, Japan, for technical assistance.

\section{Disclosure/conflict of interest}

The authors declare no conflict of interest.

\section{References}

1 Ma PC, Maulik G, Christensen J, et al. c-Met: structure, functions and potential for therapeutic inhibition. Cancer Metastasis Rev 2003;22:309-325.
2 Peruzzi B, Bottaro DP. Targeting the c-Met signaling pathway in cancer. Clin Cancer Res 2006;12:3657-3660.

3 Birchmeier C, Birchmeier W, Gherardi E, et al. Met, metastasis, motility and more. Nat Rev Mol Cell Biol 2003;4:915-925.

4 Lorenzato A, Olivero M, Patanè S, et al. Novel somatic mutations of the MET oncogene in human carcinoma metastases activating cell motility and invasion. Cancer Res 2002;62:7025-7030.

5 Tanyi J, Tory K, Rigó Jr J, et al. Evaluation of the tyrosine kinase domain of the Met proto-oncogene in sporadic ovarian carcinomas. Pathol Oncol Res 1999; 5:187-191.

6 Schmidt L, Junker K, Nakaigawa N, et al. Novel mutations of the MET proto-oncogene in papillary renal carcinomas. Oncogene 1999;18:2343-2350.

7 Kuniyasu H, Yasui W, Kitadai Y, et al. Frequent amplification of the c-met gene in scirrhous type stomach cancer. Biochem Biophys Res Commun 1992; 189:227-232.

8 Tsujimoto H, Sugihara H, Hagiwara A, et al. Amplification of growth factor receptor genes and DNA ploidy pattern in the progression of gastric cancer. Virchows Arch 1997;431:383-389.

9 Hara T, Ooi A, Kobayashi M, et al. Amplification of c-myc, K-sam, and c-met in gastric cancers: detection by fluorescence in situ hybridization. Lab Invest 1998;78:1143-1153.

10 Miller CT, Lin L, Casper AM, et al. Genomic amplification of MET with boundaries within fragile site FRA7G and upregulation of MET pathways in esophageal adenocarcinoma. Oncogene 2006;25:409-418.

11 Zhao X, Weir BA, LaFramboise T, et al. Homozygous deletions and chromosome amplifications in human lung carcinomas revealed by single nucleotide polymorphism array analysis. Cancer Res 2005;65:5561-5570.

12 Bean J, Brennan C, Shih JY, et al. MET amplification occurs with or without T790 M mutations in EGFR mutant lung tumors with acquired resistance to gefitinib or erlotinib. Proc Natl Acad Sci USA 2007; 104:20932-20937.

13 Di Renzo MF, Olivero M, Giacomini A, et al. Overexpression and amplification of the met/HGF receptor gene during the progression of colorectal cancer. Clin Cancer Res 1995;1:147-154.

14 Yamamoto S, Tsuda H, Miyai K, et al. Gene amplification and protein overexpression of MET are common events in ovarian clear-cell adenocarcinoma: Their roles in tumor progression and prognostication of the patient. Mod Pathol 2011;24:1146-1155.

15 Lee KR, Tavassoli FA, Part J, et al. Surface epithelialstromal tumours. In: Tavassoli FA, Devilee P (eds). World Health Organization Classification of Tumours. Pathology and Genetics of Tumours of the Breast and Female Genital Organs. IARC Press: Lyon, 2003, pp 117-145.

16 Chan JK, Teoh D, Hu JM, et al. Do clear cell ovarian carcinomas have poorer prognosis compared to other epithelial cell types? A study of 1411 clear cell ovarian cancers. Gynecol Oncol 2008;109:370-376.

17 Mizuno M, Kikkawa F, Shibata K, et al. Long-term follow-up and prognostic factor analysis in clear cell adenocarcinoma of the ovary. J Surg Oncol 2006; 94:138-143.

18 O’Brien ME, Schofield JB, Tan S, et al. Clear cell epithelial ovarian cancer (mesonephroid): bad prognosis only in early stages. Gynecol Oncol 1993;49:250-254. 
19 Takano M, Kikuchi Y, Yaegashi N, et al. Clear cell carcinoma of the ovary: a retrospective multicentre experience of 254 patients with complete surgical staging. Br J Cancer 2006;94:1369-1374.

20 Prowse AH, Manek S, Varma R, et al. Molecular genetic evidence that endometriosis is a precursor of ovarian cancer. Int J Cancer 2006;119:556-562.

21 Fukunaga M, Nomura K, Ishikawa E, et al. Ovarian atypical endometriosis: its close association with malignant epithelial tumours. Histopathology 1997; 30:249-255.

22 Yamamoto S, Tsuda H, Takano M, et al. Expression of platelet-derived growth factors and their receptors in ovarian clear-cell carcinoma and its putative precursors. Mod Pathol 2008;21:115-124.

23 Yamamoto S, Tsuda H, Miyai K, et al. Cumulative alterations of p27-related cell-cycle regulators in the development of endometriosis-associated ovarian clear cell adenocarcinoma. Histopathology 2010;56:740-749.

24 Yamamoto S, Tsuda H, Takano M, et al. Clear-cell adenofibroma can be a clonal precursor for clear-cell adenocarcinoma of the ovary: a possible alternative ovarian clear-cell carcinogenic pathway. J Pathol 2008;216:103-110.

25 Kurman RJ, Shih IeM. The origin and pathogenesis of epithelial ovarian cancer: a proposed unifying theory. Am J Surg Pathol 2010;34:433-443.

26 Tan DS, Kaye S. Ovarian clear cell adenocarcinoma: a continuing enigma. J Clin Pathol 2007;60:355-360.

27 Nitta H, Hauss-Wegrzyniak B, Lehrkamp M, et al. Development of automated brightfield double in situ hybridization (BDISH) application for HER2 gene and chromosome 17 centromere (CEN 17) for breast carcinomas and an assay performance comparison to manual dual color HER2 fluorescence in situ hybridization (FISH). Diagn Pathol 2008;3:41.

28 Cappuzzo F, Hirsch FR, Rossi E, et al. Epidermal growth factor receptor gene and protein and gefitinib sensitivity in non-small-cell lung cancer. J Natl Cancer Inst 2005;97:643-655.

29 Pauletti G, Dandekar S, Rong H, et al. Assessment of methods for tissue-based detection of the HER-2/neu alteration in human breast cancer: a direct comparison of fluorescence in situ hybridization and immunohistochemistry. J Clin Oncol 2000;18:3651-3664.

30 Huntsman D, Resau JH, Klineberg E, et al. Comparison of c-met expression in ovarian epithelial tumors and normal epithelia of the female reproductive tract by quantitative laser scan microscopy. Am J Pathol 1999;155:343-348.
31 Khan KN, Kitajima M, Hiraki K, et al. Immunopathogenesis of pelvic endometriosis: role of hepatocyte growth factor, macrophages and ovarian steroids. Am J Reprod Immunol 2008;60:383-404.

32 Sawada K, Radjabi AR, Shinomiya N, et al. c-Met overexpression is a prognostic factor in ovarian cancer and an effective target for inhibition of peritoneal dissemination and invasion. Cancer Res 2007;67: 1670-1679.

33 Zillhardt M, Christensen JG, Lengyel E. An orally available small-molecule inhibitor of c-Met, PF2341066, reduces tumor burden and metastasis in a preclinical model of ovarian cancer metastasis. Neoplasia 2010;12:1-10.

34 Ma PC, Jagadeeswaran R, Jagadeesh S, et al. Functional expression and mutations of c-Met and its therapeutic inhibition with SU11274 and small interfering RNA in non-small cell lung cancer. Cancer Res 2005; 65:1479-1488.

35 Ma PC, Tretiakova MS, MacKinnon AC, et al. Expression and mutational analysis of MET in human solid cancers. Genes Chromosomes Cancer 2008;47: 1025-1037.

36 Engelman JA, Zejnullahu K, Mitsudomi T, et al. MET amplification leads to gefitinib resistance in lung cancer by activating ERBB3 signaling. Science 2007; 316:1039-1043.

37 Smolen GA, Sordella R, Muir B, et al. Amplification of MET may identify a subset of cancers with extreme sensitivity to the selective tyrosine kinase inhibitor PHA-665752. Proc Natl Acad Sci USA 2006;103: 2316-2321.

38 Abounader R, Ranganathan S, Lal B, et al. Reversion of human glioblastoma malignancy by U1 small nuclear RNA/ribozyme targeting of scatter factor/hepatocyte growth factor and c-met expression. J Natl Cancer Inst 1999;91:1548-1556.

39 Matsumoto K, Nakamura T. NK4 (HGF-antagonist/ angiogenesis inhibitor) in cancer biology and therapeutics. Cancer Sci 2003;94:321-327.

40 Jun HT, Sun J, Rex K, et al. AMG 102, a fully human anti-hepatocyte growth factor/scatter factor neutralizing antibody, enhances the efficacy of temozolomide or docetaxel in U-87 MG cells and xenografts. Clin Cancer Res 2007;13:6735-6742.

41 Yamamoto S, Tsuda H, Shimazaki H, et al. Clear cell adenocarcinoma with a component of poorly differentiated histology: a poor prognostic subgroup of ovarian clear cell adenocarcinoma. Int J Gynecol Pathol 2011;30:431-441. 